Article

\title{
Effect of Diversion Cavity Geometry on the Performance of Gas-Liquid Two-Phase Mixed Transport Pump
}

\author{
Chenhao Li, Xingqi Luo, Jianjun Feng *, Guojun Zhu and Sina Yan
}

State Key Laboratory of Eco-hydraulics in Northwest Arid Region, Xi'an University of Technology, Xi'an 710048, China; drleech_xaut@126.com (C.L.); luoxq@xaut.edu.cn (X.L.); guojun_zhu1984@126.com (G.Z.); 18702970872@163.com (S.Y.)

* Correspondence: jianjunfeng@xaut.edu.cn; Tel.: +86-188-0293-6315

Received: 17 February 2020; Accepted: 6 April 2020; Published: 13 April 2020

\begin{abstract}
For the purpose of improving the transport capability of the mixed transport pump, a new self-made three-stage deep-sea multiphase pump was taken as the research object. Based on the Euler-Euler heterogeneous flow model, liquid (water) and gas (air) are used as the mixed media to study the external characteristics and internal flow identities of the mixed pump under different gas volume fraction (GVF) conditions. According to the simulation results, a local optimal design scheme of the diversion cavity in the dynamic and static connection section is proposed. The numerical results before and after the optimization are compared and analyzed to explore the effect of the diversion cavity optimization on the performance, blade load and internal flow identities of the pump. The results show that the head and efficiency are obviously improved when the inner wall of the diversion cavity is reduced by $4 \mathrm{~mm}$ along the radial direction. After optimization, under the condition of $10 \%$ gas content, the head and efficiency is increased by $3.73 \%$ and $2.91 \%$ respectively. Meanwhile, the hydraulic losses of the diversion cavity and diffuser are reduced by $9.11 \%$ and $4.32 \%$ respectively. The gas distribution in the impeller is improved obviously and the phenomenon of a large amount of gas phase accumulation is eliminated in the channel. In addition, the abnormal pressure load on the blade surface is eliminated and the turbulent flow energy intensity is reduced. The average turbulent kinetic energy $\left(\mathrm{T}_{K}\right)$ at $i=0.51$ of the first stage impeller passage is reduced by $35 \%$. Finally, the reliability of the numerical method is verified by the experimental results. To sum up, the performance and internal flow identities of the optimized mixed transport pump are improved, which verifies the availability and applicability of the optimization results. This provides a reference for the research and design of a multiphase mixed transport pump in the future.
\end{abstract}

Keywords: mixed transport pump; optimization design; diversion cavity retrofit; gas-liquid two-phase flow; numerical simulation

\section{Introduction}

Since the $21^{\text {st }}$ century, in order to obtain more fossil fuel resources, countries around the world have begun to vigorously develop equipment for deep-sea oil drilling and transportation. The mixed transport pump is mainly used in the oil industry. It is used to directly pressurize and deliver the gas-liquid mixed fluid. It can reduce production cost and simplify production management, which has great economic value and research significance [1]. However, many exploitation problems have been solved gradually under the constant research and innovation of scientists and engineers. However, many technical problems in the field of deep-sea gas-liquid transportation still need to be further studied [2]. 
This is especially the case in the deep-sea oil and gas production and transportation project; on the one hand, it can provide power for the oil and gas pipeline transportation; on the other hand, it can bring about the recovery and utilization of associated gas resources in the production process of the oil well. In order to solve the problems of low pressurization capacity and the efficiency of multi-phase mixed transport pump under high pressure and high gas volume fraction in deep-sea oil and gas production and transportation, the structure optimization design of the mixed transport pump is carried out through numerical simulation and experimental research to improve the operation performance of the pump. Moreover, the physical correlation mechanism between the external characteristics of the pump and the distribution at the gas-liquid interface is revealed.

Generally, the crude oil is a multiphase mixture of oil, gas, water, and various impurities. In order to efficiently collect and transport the mixture, a new type of equipment with the performance of both a conventional pump and compressor is required. Therefore, the multiphase mixed transport pump emerges [3,4]. As an efficient and stable production and transportation equipment, a multistage mixed transport pump is extensively used in deep-sea oil and gas transportation projects [5]. Researchers in developed countries have done a lot of research on multiphase pump technology by using numerical simulation and experimental research. Shi et al. [6] studied the load distribution characteristics of impeller blades in gas-liquid two-phase multistage oil-gas mixed transport pumps. Poullikkas [7] based on the control volume method, combined with the pump internal flow characteristics, established the performance model of the mixed transportation pump under the gas and liquid two-phase flow conditions. Caridad et al. [8] used a dual-fluid model to analyze the two-phase flow in the impeller, but neglected the influence of the interaction between the rotor and the stator. Yiming et al. [9] simulated and visualized the flow field of an electric submersible pump based on Population Balance Model (PBM), studied the effect of gas volume fraction on pump head, and found that gas accumulation and phase separation occurred on the suction surface of the impeller blade. Caridad et al. [10] used ANSYS CFX software to carry out the numerical calculation of the ESP and found that the bubble diameter has a great influence on the simulation results, and the accumulation of air on the blade surface leads to the decline of the pump performance. Barrios et al. [11] calculated the two phases flow of the ESP and researched the effect of bubble size and gas resistance coefficient on the flow characteristics of the ESP through experimental verification. Suh et al. [12] conducted numerical analysis on the axial-flow mixed transport pump under different air holdup conditions and concluded that with the increase of air holdup, the blockage of flow passage reduced the pump performance.

In recent years, with researchers' contribution to the gas-liquid mixed transport technology, the mixed transport pump technology has developed rapidly. Gamboa [13] studied dynamic multiphase flow in an electric submersible pump from both experimental and theoretical aspects and determined the effect of flow pattern and bubble size on pump performance. Huang and $\mathrm{Wu}$ [14] carried out the simulation of vane pump with the two-phase flow conditions and obtained the deviation degree of liquid-phase streamline and bubble track under different gas contents. Yu et al. [15] calculated the unsteady state of the mixed pump and found that there were discontinuous air masses with different shapes and sizes during the operation of the mixed pump. Qin [16] carried out numerical calculation for the nuclear main pump under different working gas content conditions, modified the guide vane structure, and improved the performance of the pump. Zhu [17] utilized the CFX standard $k-\varepsilon$ turbulence model to carry out the numerical calculation of gas and liquid two-phases flow in the electric submersible pump, and compared the test results, which provided help for the verification and improvement of the new bubble analysis model. Cheng et al. [18] conducted numerical calculation on the multistage centrifugal pump and obtained the detailed flow pattern inside the multistage pump; the results agree well with the experiment. Yuan et al. [19] conducted a numerical simulation of a centrifugal pump based on the non-homogeneous flow model and found that the inlet gas content has a significant effect on the performance and working stability of the pump. At present, there are many researches on the screw axial-flow multiphase pump, and the design speeds of the experimental 
prototype are mainly $1800 \mathrm{rpm}, 2000 \mathrm{rpm}$ and $3600 \mathrm{rpm}$, so the handling capacity for gas-liquid flows is limited.

From the above, most scholars' research on mixed transport pumps have mainly focused on the influence of gas relative to the internal flow pattern of the mixed transport pump. However, there are few studies on the effect of the structure optimization in a mixed transport pump on the performance of a gas-liquid two-phase mixed transport pump. Meanwhile, the influence of the diversion cavity shape change on the mixed transport pump performance is relatively less. Therefore, in this study, combined with the actual deep-sea oil and gas exploitation project, the developed deep-sea multiphase pump was taken as the research target, and the two-phase flow mathematical model of this pump was established. On this basis, the optimal design scheme of diversion cavity is proposed, and the numerical calculation is carried out for the gas-liquid two-phase operation before and after the optimization of mixed transport pump, and the reliability of the numerical method and the effectiveness of the optimal design scheme are verified by experiments. Finally, the effect of optimization on the performance and internal flow identities of this object is studied. It provides foundations for the research and design of the deep sea mixed transport pump in the future.

\section{Computational Model and Methodology}

\subsection{Physical Model Description}

The schematic diagram of the mixed transport pump prototype model studied in this paper is shown in Figure 1. The geometric structure arguments are as follows: impeller blade number is 7 , inlet diameter $\mathrm{D}_{1}=62.23 \mathrm{~mm}$, outlet diameter $\mathrm{D}_{2}=127 \mathrm{~mm}$, diffuser blade number is 10 , diffuser inlet diameter $\mathrm{D}_{3}=150 \mathrm{~mm}$, and diffuser outlet diameter $\mathrm{D}_{4}=65.49 \mathrm{~mm}$. The design parameters are: design discharge $Q=26.5 \mathrm{~m}^{3} / \mathrm{h}$, design head $\mathrm{H}=26 \mathrm{~m}$, rated efficiency of pure water $\eta=75 \%$, rated speed $n=3500 \mathrm{r} / \mathrm{min}$, and specific speed $n_{s}=98$. The computational fluid dynamics (CFD) calculation domain of the three stages mixed transport pump is shown in Figure 2. The geometric model of the mixed transport pump consists of the inlet pipe, impeller, diversion chamber, diffuser, and outlet pipe. In order to make the mixed medium entering the impeller be fully developed and to ensure the accuracy of calculation, the inlet and outlet pipes are extended appropriately.

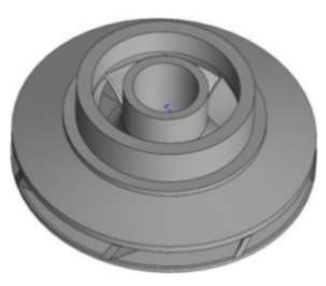

(a)

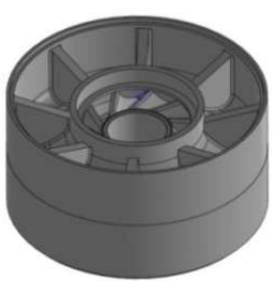

(b)

Figure 1. The geometric model. (a) Impeller; (b) Diffuser.

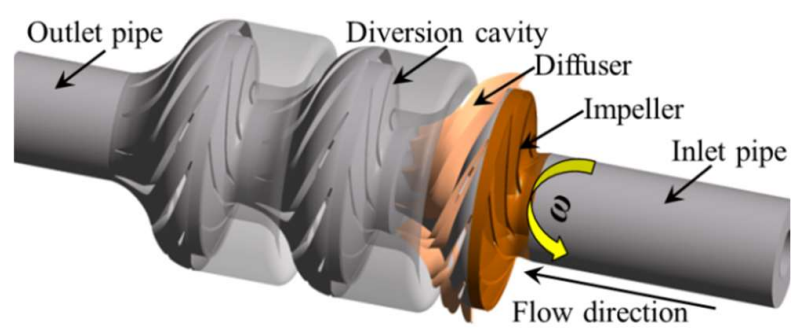

Figure 2. The CFD computational domain. 


\subsection{Mesh Generation and Turbulence Model Verification}

The grid quality is the prerequisite to ensure the accuracy of numerical simulation. The structural grid of the hexahedron has good orthogonality and the grid quality fulfills the requirements of numerical simulation. In this work, Ansys-ICEM is used to divide the hexahedral structural meshes of the main flow passing components of the mixed transport pump, moreover, the meshes of the boundary layer on the near wall and the parts with excessively large distortion are encrypted. The impeller and diffuser meshes are shown in Figure 3.

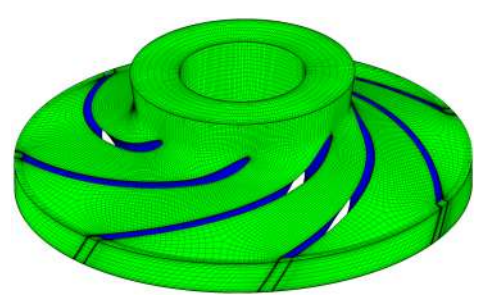

(a)

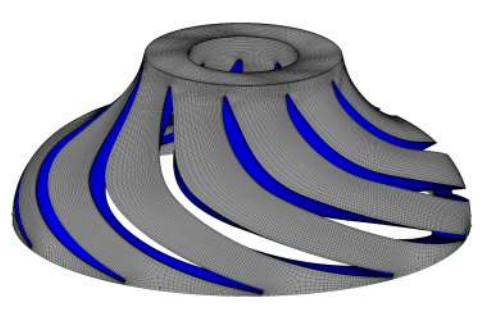

(b)

Figure 3. Mesh of main components. (a) Impeller; (b) Diffuser.

In addition, by comparing the performance curves of different grid numbers, the grid independence is verified. The calculation results are shown in Figure 4. When the grid number of the impeller and diffuser is more than 0.88 million, the head deviation is less than $1 \%$, and the average $y+$ value of the blade surface tends to be stable. Finally, the total grid number is $6,404,550$ as the numerical calculation grid.

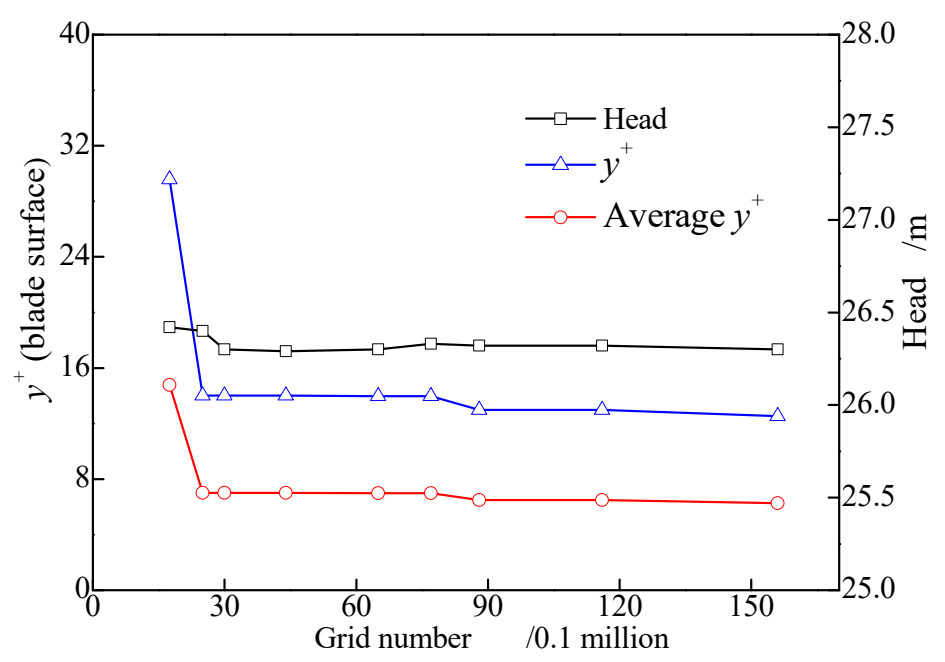

Figure 4. Mesh independence validation.

The turbulence model selection is an important work in CFD simulation and is also the key to match the calculation amount and numerical accuracy. Figure 5 shows the numerical calculation results of the mixed transport pump with different turbulence models and compared with the design value and test results. The experimental results are the two-phase flow characteristics of the three-stage mixed transport pump were tested on the multiphase mixed transport pump test bed and the data were monitored. Both the experimental devices and the measuring apparatus met the accuracy requirements. The results indicate that there is no significant difference in the predicted head variation trend between different turbulence models. However, the variation trend of head curve predicted by shear-stress transport (SST) Turbulence model is similar to the design value and experimental results, because of its advantage of capturing shear flow diffusion and separation at low Reynolds number, 
especially near the design flow area. Therefore, the SST Turbulence model is adopted for subsequent numerical simulation.

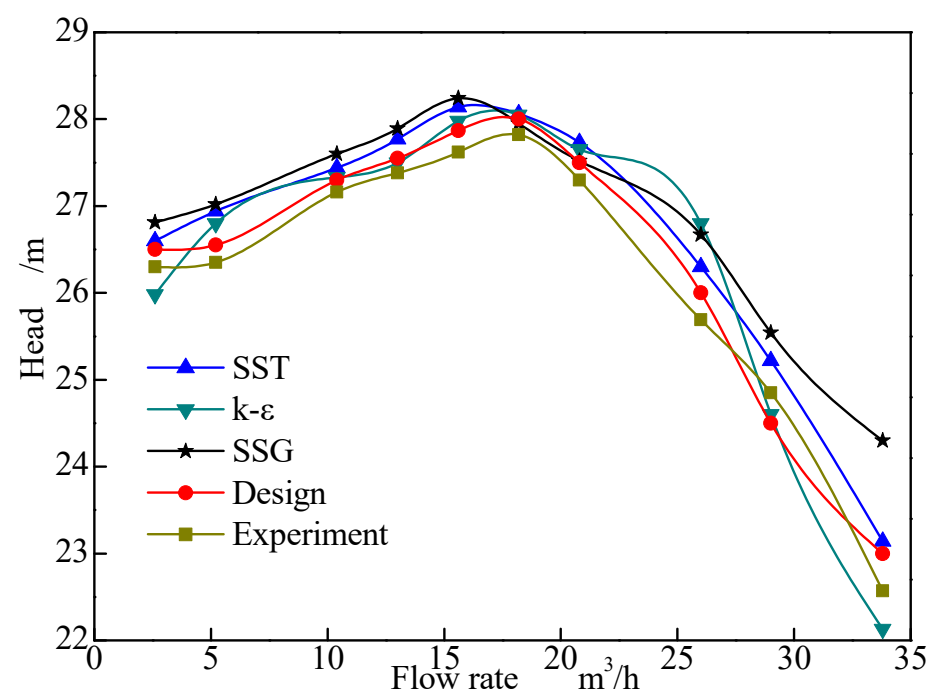

Figure 5. Turbulence models validation.

\subsection{Numerical Method}

In the numerical simulation of multiphase flow, there are two types of Eulerian-Eulerian multiphase flow models: homogeneous flow model and heterogeneous flow model. The former did not consider the velocity slip and assumed the velocity of each phase was the same; while the heterogeneous flow modal considered not only the velocity slip, but also the quality and momentum transfer of each phase [20]. In the heterogeneous flow model, continuous phase is adopted for the liquid phase, and discrete phase is adopted for the gas phase. Each phase maintains its own velocity field and temperature field; finally, the velocity and temperature of the two phases can be balanced through interphase force and heat transfer [11]. Therefore, heterogeneous flow model is adopted for numerical simulation in this paper. Without considering the influence of the temperature field, it is assumed that the gas-liquid two-phase flow is bubbly flow and meets the conservation of mass and momentum, and the particle model is adopted for the phase transfer unit. In this study, it is assumed that the flow pattern of gas-liquid two-phase flow is bubbly flow, and both the conservation of mass and momentum are satisfied, then, the mass conservation equation and momentum equation [21] are shown as follows:

The mass conservation equation and momentum conservation equation is shown as: Equations (1) and (2)

$$
\begin{gathered}
\frac{\partial}{\partial \mathrm{t}}\left(\alpha_{\mathrm{i}} \rho_{\mathrm{i}}\right)+\nabla\left(\alpha_{\mathrm{i}} \rho_{\mathrm{i}} u_{\mathrm{i}}\right)=0 \\
\frac{\partial}{\partial \mathrm{t}}\left(\alpha_{\mathrm{i}} \rho_{\mathrm{i}} u_{\mathrm{i}}\right)+\nabla\left(\alpha_{\mathrm{i}} \rho_{\mathrm{i}} u_{\mathrm{i}} \otimes u_{\mathrm{i}}\right)=-\alpha_{\mathrm{i}} \nabla P_{\mathrm{i}}+\nabla\left(\alpha_{\mathrm{i}} \mu_{\mathrm{i}}\left(\nabla u_{\mathrm{i}}+\left(\nabla u_{\mathrm{i}}\right)^{T}\right)\right)+\alpha_{\mathrm{i}} \rho_{\mathrm{i}} f_{\mathrm{i}}+M_{\mathrm{i}}
\end{gathered}
$$

where $i$ is any phase ( $l$ and $g$ represents liquid and gas, respectively), $\alpha_{\mathrm{i}}$ is volume fraction of phase $i, \rho_{\mathrm{i}}$ is the density of phase $i, u_{\mathrm{i}}$ is dynamic viscosity of phase $i, \mu_{\mathrm{i}}$ is the fluid velocity of phase $i$ in impeller channel, $f_{\mathrm{i}}$ is mass force, and $M_{\mathrm{i}}$ is the surface tension.

The relationship between the gas volume fraction and liquid volume fraction is as follows:

$$
\alpha_{\mathrm{g}}=\frac{Q_{\mathrm{a}}}{Q_{\mathrm{a}}+Q_{\mathrm{w}}}=1-\alpha_{1}
$$

where $\alpha_{\mathrm{g}}$ and $\alpha_{\mathrm{l}}$ are gas volume fraction and liquid volume fraction, respectively, and $Q_{\mathrm{a}}$ and $Q_{\mathrm{w}}$ respectively represent the volume flow of air and water. 


\subsection{Boundary Conditions and Solution Settings}

Since the flow field inside the mixed transport pump is a kind of complex turbulent flow, in this paper, the numerical simulation three-stage mixed transport pump was conducted by CFD software Ansys CFX 18.0. Euler-Euler inhomogeneous turbulence model was adopted in the calculation without considering the influence of the temperature field. The SST turbulence model was utilized for the continuous phase (water). Moreover, the discrete phase (air) taken in the dispersed phase zero equation model satisfied the mass and momentum conservation at the same time. The finite volume method was adopted for solving the governing equation discretely, and the coupling of pressure and velocity were solved by SIMPLEC method. In the numerical calculation, the interface was put as a frozen rotor mode. The other boundary conditions were as follows: the inlet was the total pressure and given the inlet gas volume fraction; meanwhile, according to the experiment dates, the mass flow rate was given at the outlet, and the no-slip wall was adopted for the physical walls surface of the mixed transport pump. The steady-state simulation convergence precision was set to a Root Mean Square (RMS) value less than $10^{-4}$.

\section{Results and Discussion}

\subsection{Optimized Size Determination}

Based on the numerical and experimental results of gas and liquid two phases flow, an optimization scheme for the diversion cavity geometry is proposed. The specific method is to reduce the length of the diversion cavity inner wall along the diameter and to reduce the gas accumulation, so as to improve the operation performance of the mixed transport pump. In this paper, eight schemes are selected for the optimization calculation, and the external characteristics are analyzed under two working conditions: $5 \%$ and $10 \%$ inlet gas content. The specific optimized dimensions are shown in Table 1. Figure 6 shows the sectional diagram of the original diversion cavity model and the optimized model after the radial reduction of $1 \mathrm{~mm}$. The optimization method of other sizes is the same as the above method.

Table 1. Optimization dimension of the diversion cavity.

\begin{tabular}{lccccccccc}
\hline Number & $\mathbf{1}$ & $\mathbf{2}$ & $\mathbf{3}$ & $\mathbf{4}$ & $\mathbf{5}$ & $\mathbf{6}$ & $\mathbf{7}$ & $\mathbf{8}$ & $\mathbf{9}$ \\
\hline Size (b) & $0 \mathrm{~mm}$ & $1 \mathrm{~mm}$ & $2 \mathrm{~mm}$ & $3.5 \mathrm{~mm}$ & $3.75 \mathrm{~mm}$ & $4 \mathrm{~mm}$ & $4.25 \mathrm{~mm}$ & $4.5 \mathrm{~mm}$ & $5 \mathrm{~mm}$ \\
\hline
\end{tabular}
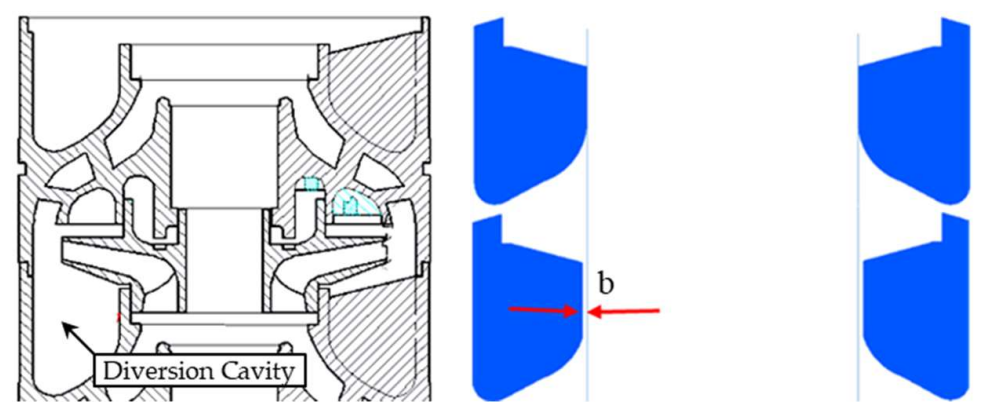

Figure 6. Structure diagram of diversion cavity and optimization schematic diagram.

The numerical simulation with different GVF was carried out of the pump, and the head and efficiency data were statistically analyzed. Efficiency $\eta$ is defined as the ratio of the actual pump head $H_{\mathrm{a}}$ and the theoretical calculation head $H_{\mathrm{t}}$. The expression is defined as follows:

$$
\eta=H_{\mathrm{a}} / H_{\mathrm{t}}
$$


where the actual pump head $H_{\mathrm{a}}$ and the theoretical head $H_{\mathrm{t}}$ are defined as:

$$
\begin{gathered}
H_{\mathrm{a}}=\left(P_{\text {out }}-P_{\text {in }}\right) /\left(\rho_{\text {mix }} \cdot \mathrm{g}\right) \\
H_{\mathrm{t}}=(M \times \omega) /\left(\rho_{\text {mix }} \cdot \mathrm{Q} \cdot \mathrm{g}\right) \\
\rho_{\text {mix }}=G V F \times \rho_{\text {gas }}+(1-G V F) \times \rho_{\text {water }} G V F=\alpha_{g}
\end{gathered}
$$

where $P_{\text {out }}$ and $P_{\text {in }}$ represent the outlet pressure and the inlet pressure of mixed transport pump, respectively; $\rho_{\text {mix }}, \rho_{\text {gas }}$, and $\rho_{\text {water }}$ are the gas-liquid mixed density, the gas density, and the liquid density, respectively; g represents gravity acceleration; and $Q$ represents volume flow rate.

The effects of different optimized sizes on the head and efficiency of the mixed transport pump are shown in Figures 7 and 8 . As we can see, under the 5\% inlet GVF, the performance curves changes little with diversion cavity size and the curve fluctuates little. However, the mixed transport pump head and efficiency enhance gradually with the increase of the optimal size under the $10 \%$ inlet GVF. When the optimal size reaches $4 \mathrm{~mm}$, the pump head and efficiency reach the maximum value. Based on the external characteristics analysis, it is determined that the diversion cavity inner wall was reduced by $4 \mathrm{~mm}$ and is the best along the radial direction. Especially, the optimized model head and efficiency respectively increased $2.5 \mathrm{~m}$ and $4.11 \%$ more than the original model under $10 \%$ inlet GVF. Finally, the optimized size of the diversion cavity was determined to be $4 \mathrm{~mm}$.

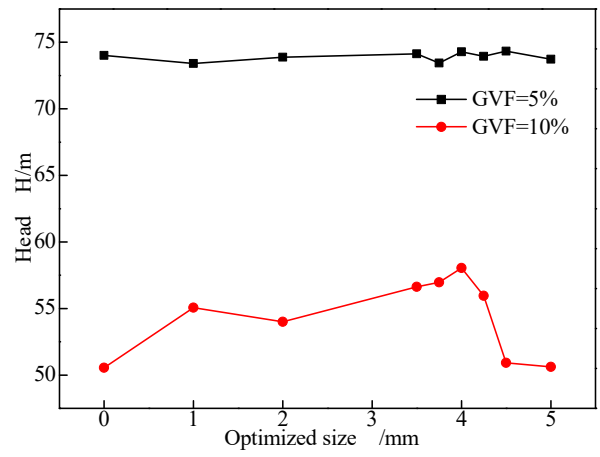

Figure 7. Head curve of different optimized sizes.

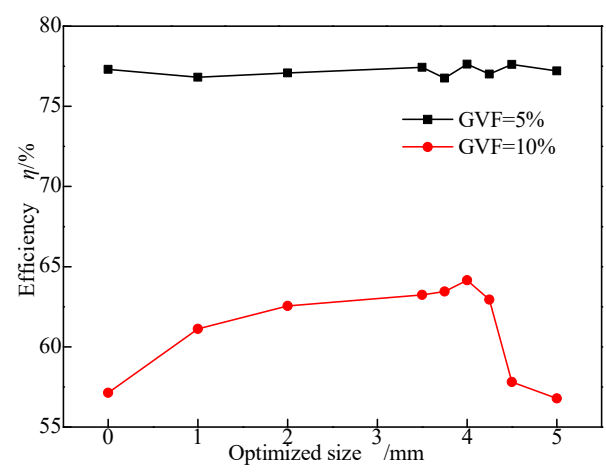

Figure 8. Efficiency curve of different optimized sizes.

\subsection{Influence of Optimization on Blade Loading}

Figure 9 shows the load distribution curve of the impeller blade before and after the optimization under the design condition with different air contents. In this figure, $\mathrm{t} / \mathrm{T}$ is defined as the relative position of a point on the blade on the whole blade surface, and the value range of the blade is $0-1$ (where $t$ is the distance from the blade head to the load interception point, and $\mathrm{T}$ is the blade total length, $\mathrm{mm}$ ), and the vertical coordinate represents the pressure distribution on the blade surface. It can be seen that due to less gas accumulation in the flow passage and smooth flow passage, the blade 
load change trends before and after the optimization is basically the same under the $5 \%$ air content condition. After optimization, the blade load is slightly higher than the original model and the pressure load on the blade front and back is evenly distributed. When GVF $=10 \%$, the blade load changes greatly before and after the optimization. Before the optimization, the suction surface load is larger than the pressure surface in the range from the blade head to $1 / 3$ position, which is caused by the gas gathering at the suction side of the blade head, resulting in the high pressure. After optimization, the load distribution on the blade surface is uniform, which improves the abnormal load distribution and the performance of the impeller.

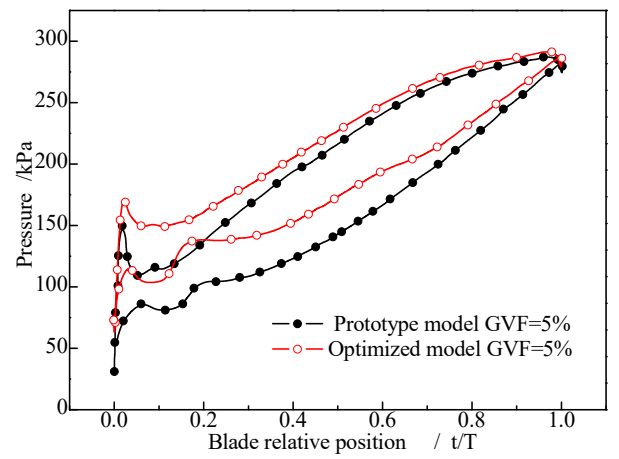

(a)

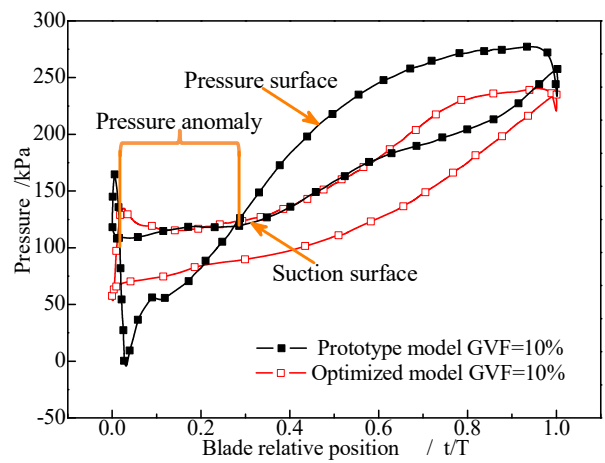

(b)

Figure 9. Impeller blade load distribution. (a) GVF $=5 \%$; (b) GVF $=10 \%$.

\subsection{Effect of Optimization on Internal Flow Field and Performance}

\subsubsection{Influence on Gas Distribution}

In this section, the gas distribution in each stage impeller of the three-stage mixed transport pump before and after the modification and optimization of the diversion cavity is analyzed. This is due to the gas-liquid two-phase flow phenomenon in the mixed transport pump not being particularly obvious when the gas content is less than $5 \%$. However, when the gas volume fraction is more than $10 \%$, the performance of the mixed transport pump decreases sharply, and the numerical simulation cannot accurately reflect the gas accumulation phenomenon in the impeller. Therefore, the calculation results under the condition of $5 \%$ and $10 \%$ inlet gas content are taken as the analysis object. Figure 10 shows the distribution of the gas phase in impellers at all levels before and after optimization when $\mathrm{GVF}=5 \%$. It can be seen from the figure that before the modification and optimization, there is a relatively obvious gas phase accumulation in some passages of the first stage impeller, which seriously influences the performance and operation stability of the first stage impeller. With the increase of the number of stages, the gas accumulation in the impeller is gradually weakened. This is due to the fact that the pressure inside the impeller rises, the gas is compressed and the volume fraction of the gas decreases, so it is difficult to form a bubble cluster. After the modification and optimization, the gas accumulation phenomenon in the first stage impeller channel is improved obviously, there is no large gas accumulation in the channel, and the gas phase distribution in each channel is relatively balanced. At the meantime, as the stage number increases, the gas distribution is more and more uniform. 


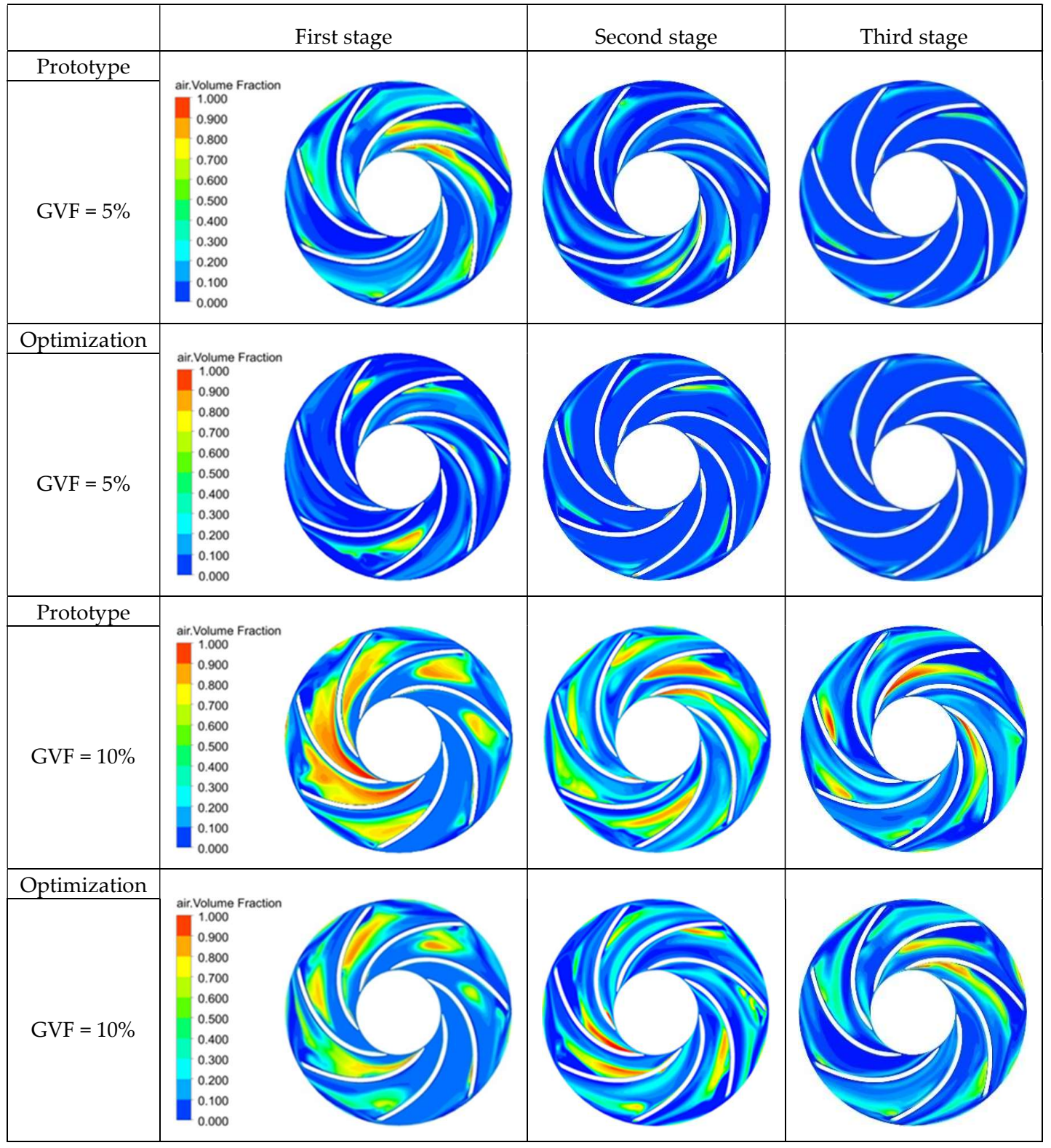

Figure 10. Gas distribution at different inlet gas volume fractions of each impeller.

\subsubsection{Effect on Pressurization and External Characteristics}

Figure 11 shows the pressure variation at each pump outlet section of the three-stage mixed transport pump before and after optimization with different inlet gas content conditions. According to the results in the analysis chart, before the optimization, the pressurization capacity at each stage pump is basically consistent under the $5 \%$ inlet gas volume fraction condition, and the value of pressure rise is about $230 \mathrm{kPa}$, indicating that the pump performance is not affected by gas under the low gas volume fraction condition. When the inlet gas content is $10 \%$, the supercharging value of the first stage pump is only $132.33 \mathrm{kPa}$ before optimization, and the supercharging capacity of the first stage pump is $33 \%$ lower than the last two stages. When the inlet gas content reaches $20 \%$, the supercharging capacity of the first two stages of this pump is greatly reduced before optimization, and the supercharging value is reduced by $37.7 \%$, while the supercharging capacity of the third stage pump is greatly improved. This is due to the fact that after the supercharging of the first two stages, the gas volume fraction is reduced, the gas aggregation level is relatively small, and the supercharging capacity of the third stage pump is improved. In addition, as the gas content increases, the supercharging capacity of the first two stages 
pump is greatly affected, which shows that the pump supercharging ability is closely related to the gas volume fraction.

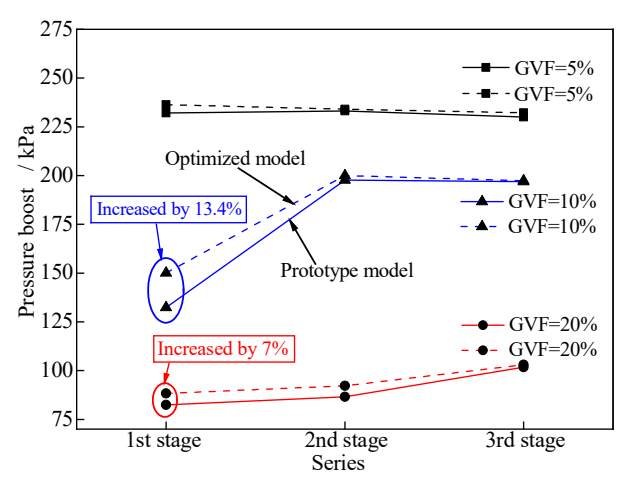

Figure 11. Pressurization of pump at different stages with different GVF.

After optimization, the supercharging capacity of all stages pump was slightly improved and remains unchanged at 5\% inlet GVF. Then when the inlet GVF was $10 \%$ and $20 \%$, the supercharging capacity of the first two-stage pump was significantly improved. Especially, the supercharging capacity of the first stage pump was increased by $13.4 \%$ and $7 \%$ respectively with the previous optimization. Meanwhile, the booster change law of each stage pump is the same as before optimization, which indicates that this optimization work does not change the overall operation state of the pump and improves the pressurization capacity of the first stage pump effectively under the high gas content condition.

Figure 12 shows the characteristic curves comparison results of the mixed transport pump before and after optimization at the design point under different inlet GVF conditions.

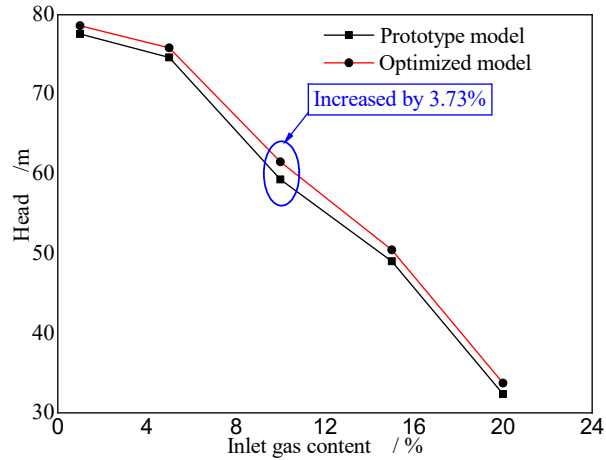

(a)

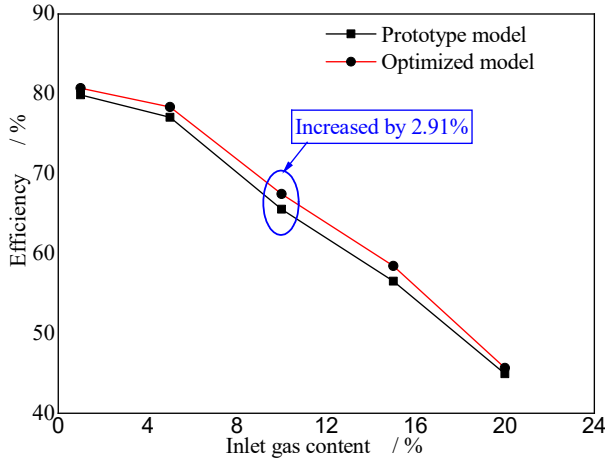

(b)

Figure 12. Performance comparison of the mixed transport pump before and after optimization. (a) Head comparison; (b) Efficiency comparison.

The analysis results of above figures show that under low gas content GVF $=5 \%$, the external characteristics of the mixed transportation pump show a good result; however, when the gas content was more than $10 \%$, the external characteristics of the pump dropped rapidly. That is due to the fact that the gas and liquid two-phases flow in the impeller is relatively stable when the gas-content is low, but when the GVF continues to increase, the interaction between the air and water intensifies, and a large amount of gas accumulates in the passage, causing the pump head to drop. When the gas content reaches $20 \%$, the flow state inside the pump becomes more disordered, while a great deal of gas in the impeller passage causes the "gas blockage" phenomenon and the pump performance also decreases significantly. However, after optimization, the head and efficiency of the three-stage mixed transport 
pump have improved to some extent. Compared with other working conditions, when the gas content was $10 \%$, the head and efficiency was significantly improved $3.73 \%$ and $2.91 \%$, respectively.

\subsection{Optimization Cause of Pump Performance Improvement}

In this section, the hydraulic loss of the main flow passage components before and after the optimization is analyzed to explore the reasons for the performance improvement of the mixed-transport pump. The hydraulic losses of the diversion cavity, as a component of the optimized modification, affect the optimization result. The calculation formula of the hydraulic loss in the diversion cavity $\left(H_{\mathrm{dc}}\right)$ is as follows:

$$
H_{\mathrm{dc}}=\frac{P_{\mathrm{dc} 1}-P_{\mathrm{dc} 2}}{\rho_{\mathrm{mix}} g}
$$

where $P_{\mathrm{dc} 1}$ and $P_{\mathrm{dc} 2}$ represent the total pressure of the outlet and inlet total of the diversion cavity, $(\mathrm{Pa})$.

Figure 13 shows the comparison diagram of hydraulic loss in main components before and after optimization under different GVF conditions. The analysis results show that the hydraulic loss of each component decreases correspondingly after the optimization, thus the hydraulic performance of this three-stage mixed transport pump is improved. In particular, under the GVF $=10 \%$ condition, the hydraulic loss of the diversion cavity and diffuser was reduced by $9.11 \%$ and $4.32 \%$, respectively, which verified the effectiveness and reliability of the optimization. Since the diffuser is a fixed part and its structure did not change before and after optimization, the decreased value of hydraulic loss is smaller than the diversion cavity.

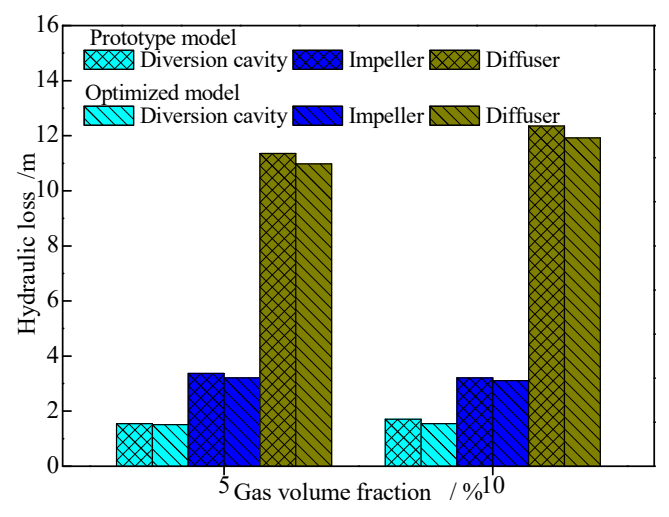

Figure 13. Hydraulic loss diagram with different gas volume fractions.

\subsection{Turbulent Kinetic Energy Distribution in Impeller}

In this section, the turbulent kinetic energy distribution in the impeller before and after the optimization is analyzed. Turbulence kinetic energy $\left(\mathrm{T}_{K}\right)$ is a representation of turbulence intensity in a turbulence model and an important embodiment of fluid and state. A single passage of impeller is selected as the research object to analyze the turbulent kinetic energy. The entire impeller passage is divided into seven sections from the inlet to the outlet, and the specific location of the section is shown in Table 2. The partitioning method is used to express the total distance from the passage inlet to the outlet as $\mathrm{L}$, and the distance from the section to the inlet is expressed in $1 . i$ is defined as the ratio of the $l$ to the $\mathrm{L}$, which is a dimensionless number. The equation is as follows:

$$
i=\frac{l}{L}
$$

Figure 14a shows the schematic diagram of the turbulent kinetic energy $\left(\mathrm{T}_{\mathrm{K}}\right)$ distribution and the section position in the passage. The $T_{K}$ gradually enhances from the passage inlet to the outlet and the range expands. Figure $14 \mathrm{~b}-\mathrm{d}$ shows the variation tendency of the average turbulent kinetic energy along the flow channel at the impeller different sections before and after the optimization under 
the $10 \%$ inlet gas content condition. The analysis results show that the turbulent kinetic energy of all stage pumps enhances gradually along the flow orientation, indicating that the gas and liquid two-phases mixes and interacts with each other in the whole passage, which impacts on the hydraulic performance of the mixed transport pump. However, the $\mathrm{T}_{\mathrm{K}}$ at the inlet and outlet of second and third stage impeller are affected by the rotor and stator interference, and the $T_{K}$ increased greatly due to the sudden change of flow pattern and attains the maximum value at the exit. With the increase of the series, because of the pressure and GVF increases, the $T_{K}$ in the impeller decreases gradually, that is, the turbulence intensity decreases. Therefore, the flow pattern in the impeller can be improved.

Table 2. Cross-section number and position relative value correspondence relationship.

\begin{tabular}{cccccccc}
\hline Number & $\mathbf{1}$ & $\mathbf{2}$ & $\mathbf{3}$ & $\mathbf{4}$ & $\mathbf{5}$ & $\mathbf{6}$ & $\mathbf{7}$ \\
\hline location & inlet & $i=0.17$ & $i=0.34$ & $i=0.51$ & $i=0.68$ & $i=0.84$ & outlet \\
\hline
\end{tabular}

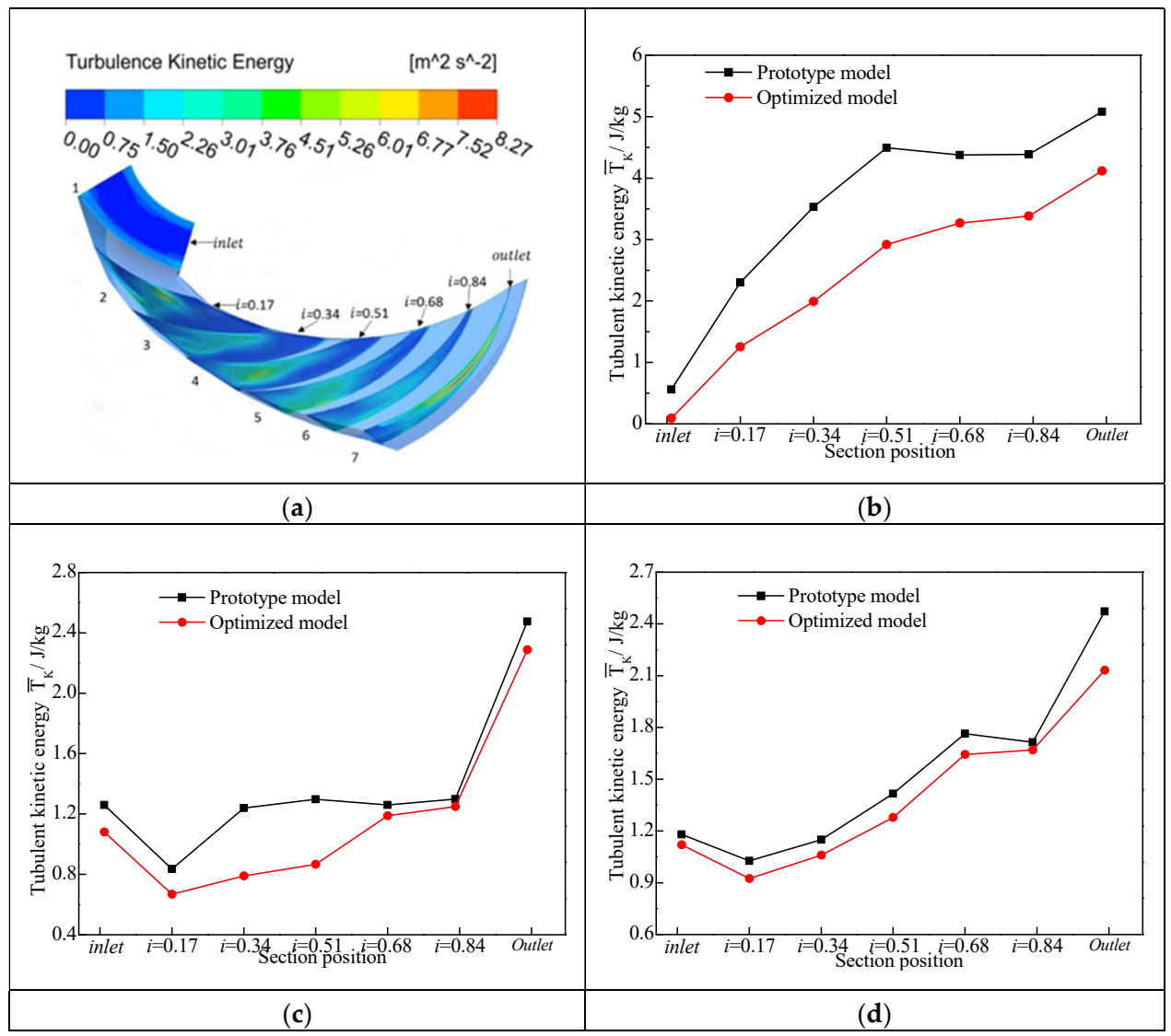

Figure 14. Distribution of average turbulent kinetic energy at $10 \%$ inlet gas volume fraction. (a) Cross section position; (b) First stage; (c) Second stage; (d) Third stage.

Compared with the same stage, the turbulent kinetic energy of the optimized mixed-transport pump decreased to a certain extent, and the $\mathrm{T}_{K}$ of the first stage impeller decreased the most obviously. The drop-out value was the largest at the passage $i=0.51$, and the $\mathrm{T}_{K}$ decreased by $35 \%$. After optimization, the effect of the gas phase on $\mathrm{T}_{K}$ weakens after the second stage impeller passage $i=0.68$; the $\mathrm{T}_{K}$ decrease value is basically a stable value, indicating that the influence of gas on the mixed transport pump is weakened and the performance is stable. Therefore, it can be predicted that the operation characteristics of this multistage pump are consistent after the third stage pump under the two phases flow conditions. 


\section{Experiment and Verification}

Figure 15 shows the principle scheme of the experimental system. The system consists of a gas-phase line, a liquid-phase line and a gas-liquid mixture line. In the experiment, the gas phase enters the buffer tank after passing through the compressor, and then enters the mixer after passing through the gas mass flow-meter. While the liquid flow rate is provided by the plunger pump and measured through the liquid mass flow-meter. After the liquid phase entering the mixer and mixing with the gas phase, finally entering the inlet of the test section of the mixed transport pump. The mixed media after the mixed transport pump is returned to the gas-liquid separator through the pipeline. In the test, data were collected by the collector to the computer for real-time recording. Both the experimental devices and the measuring apparatus met the secondary accuracy requirements of GB/T 3216-2016. By adjusting the flow rate through the outlet valve, the external characteristics of this pump under diverse flow conditions were obtained. In the multiphase flow test, the water flow rate was controlled to an invariable value, and the gas phase control valve was adjusted to control the gas flow rate into the pipeline, so as to obtain the pump external characteristics curve under different inlet gas content conditions. In the experiment, the accuracy of each measuring instrument was less than $1 \%$, and the test uncertainty was $0.32 \%$. Figure 16 is the site layout of the mixed transport pump test bed.

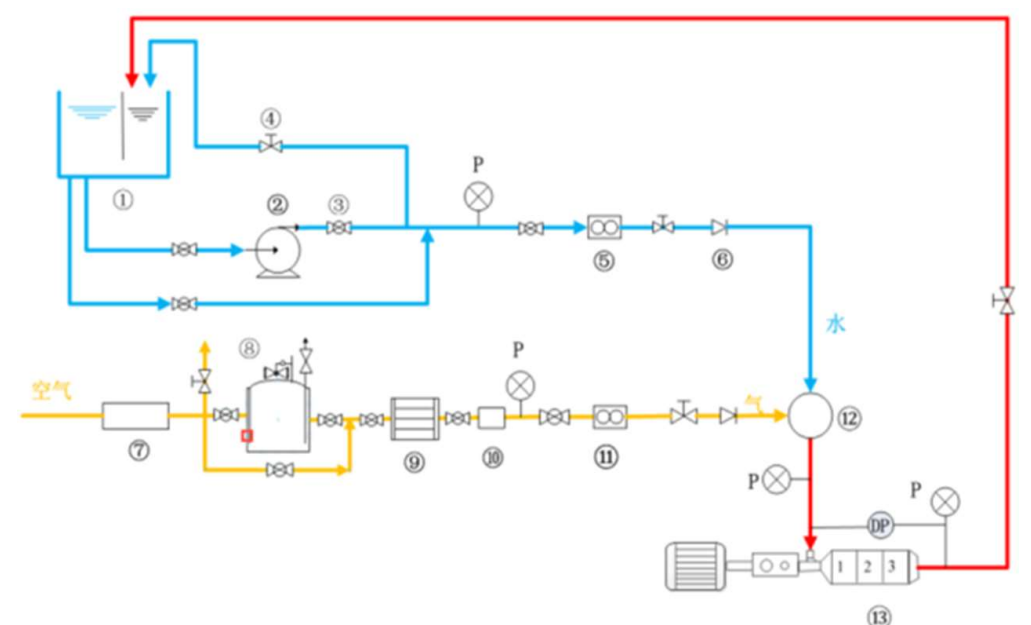

Figure 15. Experimental system principle scheme. (1) Tank; (2) Plunger pump; $(3,4)$ Valves; $(\mathbf{5 , 1 1})$ Flowmeter; (6) Check valve; (7) Air compressor; (8) Gasholder; (9) Air dryer; (10) Filter; (12) Commingler; (13) Pump Test section. Label: P: Pressure sensor; DP: Differential pressure sensor.

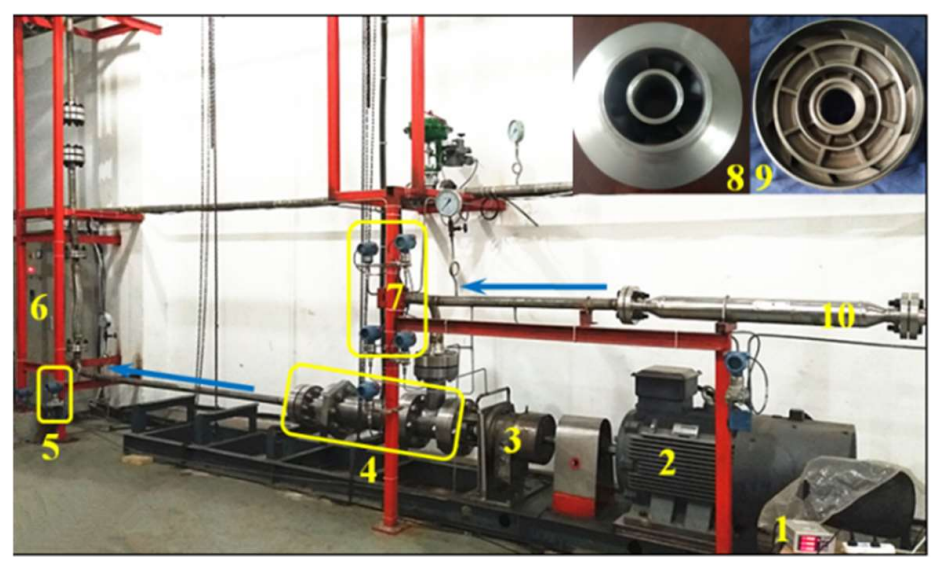

Figure 16. Site layout of the multiphase pump test bed. (1) Torque and speed displayer, (2) Electric motor, (3) Bearing housing, (4) Multiphase pump test section, (5) Differential pressure sensor, (6) Frequency converter control cabinet, (7) Pressure sensor, (8) Impeller, (9) Diffuser, (10) Mixer. 
Figures 17 and 18 shows the comparison between the numerical results of pure liquid, gas-liquid two phases and the experimental results respectively. It can be seen from the figure that the calculation consequences are in great accordance with the experimental results, which verifies the reliability and accuracy of the simulation method. The numerical calculation results are slightly higher than the experimental results, and the reason for the deviation is that the wall roughness is not considered and the leakage loss and local hydraulic loss at the impeller outlet are ignored in the numerical simulation. Under the design condition, the relative error of the head of the pump is within the allowable range under different gas contents. When the gas content is more than $15 \%$, the maximum relative error between numerical simulation and experimental value is $4.57 \%$.

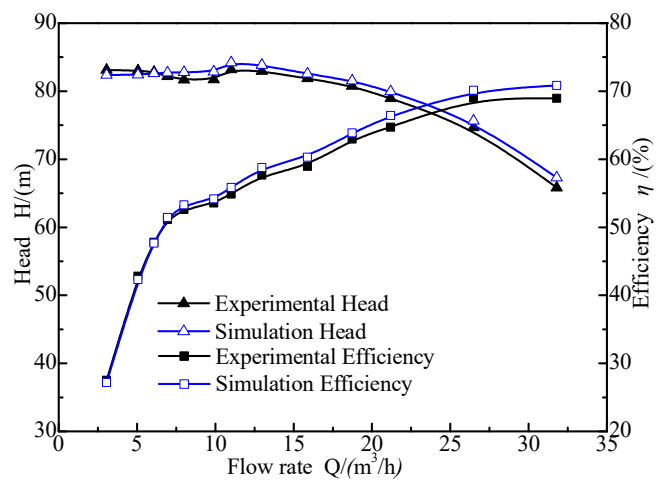

Figure 17. Performance under pure water condition.

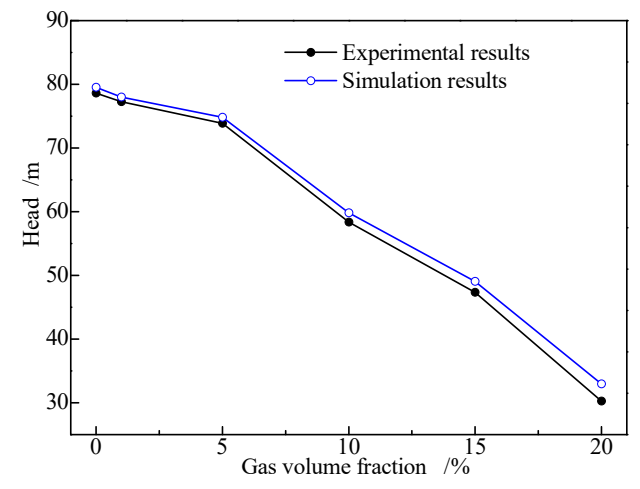

Figure 18. Comparison of simulation with experimental results under different GVFs.

\section{Conclusions}

Based on the numerical simulation and experimental study on the structure optimization of the diversion cavity of the three-stage mixed transport pump, an optimization method for the diversion cavity is proposed in this paper. After optimization, the blade load distribution is more uniform, the gas accumulation in the impeller is improved, the pressurization ability of the pump is improved, and the average turbulent kinetic energy in the impeller is reduced. These show that the optimization is effective and the main conclusions are as follows:

(1) The results of computational fluid dynamics and experiments indicate that the proposed optimization scheme is effective. When the optimized size of the inner wall of the diversion cavity was determined to be $4 \mathrm{~mm}$, at the design point, the performance of the optimized mixed transport pump was improved by $3.73 \%$ head and $2.91 \%$ in efficiency.

(2) The optimized numerical simulation results show that the abnormal pressure load of the impeller blade can be corrected, the blade pressure distribution is uniform, and the hydraulic performance of the impeller is improved, when the inlet gas volume fraction is $10 \%$. Meanwhile, the hydraulic losses of the main flow passage parts are weakened, especially the hydraulic losses of the 
diversion cavity and the diffuser are reduced by $9.11 \%$ and $4.32 \%$ respectively. The feasibility and availability of this optimization are verified.

(3) Analysis of flow field shows that the gas accumulation in each stage impeller is improved after optimization, especially in the first stage impeller; the "gas blockage" phenomenon is obviously improved; and the gas phase in each channel is evenly distributed. The transport capacity of gas and liquid two-phase flow in the impeller can be improved.

(4) The optimization results show that the turbulence energy intensity in each impeller diminishes, and the $T_{K}$ of the first stage impeller decreases most obviously. The average $T_{K}$ decreases by $35 \%$. The second impeller turbulence kinetic energy is weakened after $i=0.68$. It can be predicted that the operation characteristics of each stage are consistent after the second stage pump under the multi-phases flow conditions.

\section{Research Prospect}

In this paper, the modification optimization only focuses on the internal surface size of the diversion cavity model near the axis side, and the other structures of this pump have not been modified. In the future, the optimization method can be combined with the impeller and diffuser optimization of the mixed transport pump for further study.

Author Contributions: Investigation, numerical simulation, data analysis and paper writing, are carried out by C.L., X.L. and J.F. conceived and supervised the study and edited the manuscript. G.Z. and S.Y. also wrote the manuscript. All authors reviewed the manuscript. All authors have read and agreed to the published version of the manuscript.

Funding: This research was funded by Xingqi Luo, the National Key R\&D Program of China (No. 2018YFB1501900), the National Natural Science Foundation of China (Nos. 51527808, 51679195, 51769012 and 51909212), the Natural Science Basic Research Plan in Shaanxi Province of China (No. 2019JQ-044) and Guojun Zhu, the Scientific Research Plan Projects of Shaanxi Education Department (No. 19JK0570).

Conflicts of Interest: The authors declare no conflict of interest.

\section{References}

1. Li, F.X. Innovative application of multiphase transport pump. Int. Combust. Engine 2019, 5, 203-204.

2. Seung, H.K.; ohn, C.H.; Hwang, J.Y. Effects of tube diameter and submergence ratio on bubble pattern and performance of air-lift pump. Int. J. Multiph. Flow 2014, 58, 195-204.

3. Li, S.H.; Cao, F.; Xing, Z.W. Research and Application of Subsea Multiphase Pump. Fluid Mach. 2011, 39, $40-44$.

4. Serena, A.; Bakken, L.E. Flow Visualization of Unsteady and Transient Phenomena in a Mixed-Flow Multiphase Pump. In Proceedings of the ASME Turbo-machinery Technical Conference and Exposition, Seoul, Korea, 13-17 June 2016.

5. Zhu, J.; Guo, X.; Liang, F.; Zhang, H.-Q. Experimental study and mechanistic modeling of pressure surging in electrical submersible pump. J. Nat. Gas Sci. Eng. 2017, 45, 625-636. [CrossRef]

6. Shi, G.T.; Luo, K.; Wang, Z.W.; Liu, Y. Study on Load Distribution Characteristics of Axial Flow Multiphase Pump. Water Resour. Power 2018, 36, 175-178.

7. Poullikkas, A. Two phase flow performance of nuclear reactor cooling pumps. Prog. Nucl. Energy 2000, 36, 123-130. [CrossRef]

8. Caridad, J.; Asuaje, M.; Kenyery, F.; Tremante, A.; Aguillón, O. Characterization of a centrifugal pump impeller under two-phase flow conditions. J. Pet. Sci. Eng. 2008, 63, 18-22. [CrossRef]

9. Chen, Y.; Patil, A.; Chen, Y.; Bai, C.; Wang, Y.; Morrison, G. Numerical Study on the First Stage Head Degradation in an Electrical Submersible Pump with Population Balance Model. J. Energy Resour. Technol. 2018, 141, 022003. [CrossRef]

10. Caridad, J.; Kenyery, F. CFD Analysis of Electric Submersible Pumps (ESP) Handling Two-Phase Mixtures. J. Energy Resour. Technol. 2004, 129, 99-104. [CrossRef] 
11. Barrios, L.; Prado, M.G.; Kenyery, F. CFD Modeling Inside An Electrical Submersible Pump In Two-Phase Flow Condition. In Proceedings of the ASME 2009 Fluids Engineering Division Summer Meeting, Vail, CO, USA, 2-6 August 2009; pp. 1-13, 457-469.

12. Suh, J.-W.; Kim, J.-W.; Choi, Y.-S.; Kim, J.-H.; Joo, W.-G.; Lee, K.-Y. Development of numerical Eulerian-Eulerian models for simulating multiphase pumps. J. Pet. Sci. Eng. 2018, 162, 588-601. [CrossRef]

13. Gamboa, J. Prediction of the Transition in Two-Phase Performance of An Electrical Submersible Pump; University of Tulsa: Tulsa, OK, USA, 2009.

14. Huang, S.; Wu, Y.L. 3-D calculation of gas-oil flow in rotodynamic pump based on a bubbly flow model. J. Hydraul. Eng. 2001, 6, 57-61.

15. Yu, Z.Y.; Cao, S.L.; Wang, G.Y. A Numerical Model for 3-D Gas-Liquid Two-Phase Flow in Vane Pumps. Trans. Beijing Inst. Technol. 2007, 27, 1057-1064.

16. Qin, J. Design of Hydraulic Model and Simulation of Inner Flow Field of Nuclear Main Pump; Dalian University of Technology: Dalian, China, 2010.

17. Zhu, J.J.; Zhang, H.Q. Numerical Study on Electrical-Submersible-Pump Two-Phase Performance and Bubble-Size Modeling. Soc. Pet. Eng. 2017, 32, 267-278. [CrossRef]

18. Cheng, X.W.; Cai, G.J.; Liang, J. Three dimensional flow field simulation of multistage centrifugal pump. Pump Technol. 2017, 4, 23-31.

19. Yuan, J.P.; Zhang, K.Y.; Si, Q.R. Numerical Investigation of Gas-Liquid Two-phase Flow in Centrifugal Pumps Based on Inhomogeneous Model. Trans. Chin. Soc. Agric. 2017, 48, 89-95.

20. Fu, Q.; Xi, Y.; Zhu, R.S.; Yuan, S.Q.; Wang, X.L. AP1000nuclear main pump internal unsteady analysis under gas-liquid two phase condition. J. Vib. Shock 2015, 34, 132-136.

21. Zhou, S.Q.; Kong, F.Y.; Wang, Z.Q.; Yi, C.; Zhang, Y. Numerical simulation for low specific-speed centrifugal pump with structured grid. Trans. Chin. Soc. Agric. Mach. 2011, 42, 66-69.

(C) 2020 by the authors. Licensee MDPI, Basel, Switzerland. This article is an open access article distributed under the terms and conditions of the Creative Commons Attribution (CC BY) license (http://creativecommons.org/licenses/by/4.0/). 